\title{
Chylous Lymphatic Cyst in a Child-A Rare Variant of Mesenteric Cyst
}

\author{
Aditya Pratap Singh*, Arun Kumar Gupta, Maryem Ansari, Ramesh Tanger \\ Department of Pediatric Surgery, S.M.S. Medical College and Group of Hospitals, Jaipur, Rajasthan, India.
}

\begin{abstract}
Chylous lymphatic cysts are the rarest variety of the mesenteric cyst. These are the lymph containing cysts. The underlying pathology is benign proliferations of the lymph vessels due to obstruction in the lymphatic system. These have a variable clinical presentation. We are presenting here a case of a chylous mesenteric cyst in a 5 years old male child with acute intestinal obstruction.
\end{abstract}

Keywords: Mesenteric cyst, Chylous cyst, Small bowel, Obstruction, Lump, Excision, Recurrence.

\section{INTRODUCTION}

Chylous lymphatic cysts are the rare variant of the mesenteric cysts. These are makeup to $7.3 \%$ to $9.5 \%$ of all abdominal cysts [1] and $3 \%$ to $9.2 \%$ of all pediatric lymphangiomas [2]. Mesenteric cysts are classified into embryonic, traumatic, neoplastic, infective and degenerative. It is based on the etiology of the cysts. Mennemeyer and Smith proposed a clinically accepted classification of the mesenteric cysts. It is based on the histopathological features of the cysts. Mesenteric cysts have been reported frequently in the literature while the chylous variant is rare in the pediatric population [1]. Chylous mesenteric cysts are rare in the mesenteric cysts. It is the histopathological diagnosis. We are presenting this case here because it is rare and with histopathological features.

\section{CASE REPORT}

A 5 years old male child presented to us with the complaints of bilious vomiting and abdominal distension for last four days. There was no past history of similar complaints. Abdomen was distended and soft. Patient had not passed motion for last 3 days. Routine blood investigations were within normal limits including complete blood counts, renal function test and serum electrolytes. X-ray abdomen showed multiple air fluid levels. We presumed acute intestinal obstruction so planned for surgical exploration. We didn't performed ultrasonography and computed tomography, because there was bilious vomiting with multiple air fluid level on X-ray abdomen. We explored the patient in emergency. On exploration, there was a large multilobed swelling arising from the mesenteric side of the ileum. It was located $20 \mathrm{~cm}$ proximal to ileocaecal junction. It was compressing the adjacent ileum, so there was proximal dilatation and distal collapse bowel. There was also volvulus, but the vascularity was maintained. We corrected the volvulus and excised the swelling with the adjacent ileum

*Address correspondence to this author at the Department of Pediatric Surgery, S.M.S. Medical College and Group of Hospitals, Jaipur, Rajasthan, India. E-mail:dr.adisms@gmail.com and end to end ileo-ileal anastomosis was done. Swelling was single multilocular of size around $10 \mathrm{~cm}$ in diameter and had serous and milky fluid (Fig. 1).

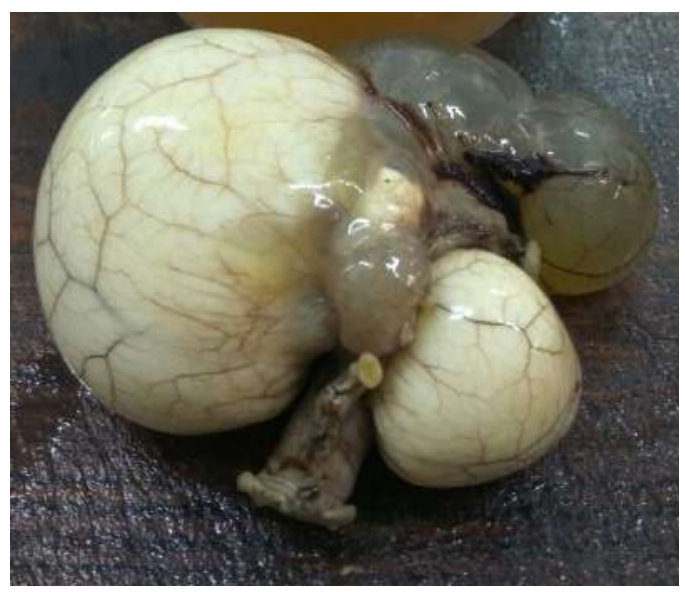

Fig. (1). Photo Showing Chylous Cyst.

Histopathology showed cyst wall with transparent fluid reveal loose fibrovascular tissue lined by flattened mesothelial cells and cyst wall containing whitish fluid revealed loose fibrocollagenous tissue along with lymphocytes (Fig. 2).
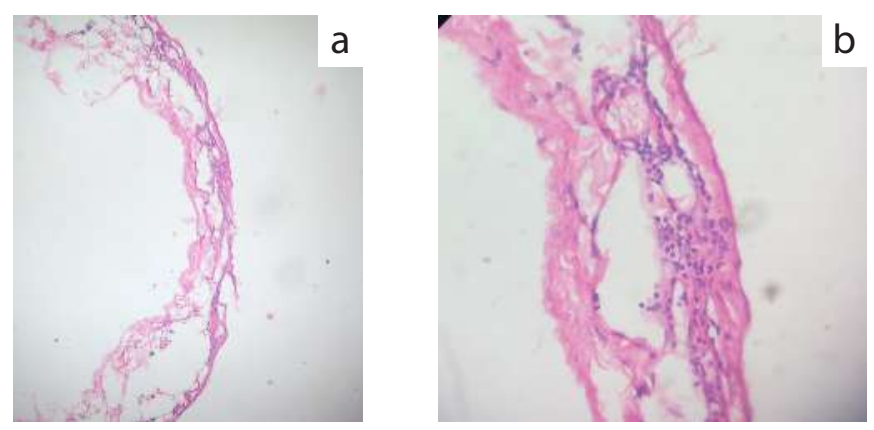

Fig. (2). Histopathology Slides.

Overall, histopathological examination was suggestive of mesenteric cyst along with chylous cyst. The patient had an uneventful postoperative recovery period. He was discharged 
7 days later after full recovery.

\section{DISCUSSION}

There are only few cases of the chylous variant of the mesenteric cysts have been reported in the literature [1]. These are resulted from an imbalance between the inflow and outflow of the fluid. Mesenteric cysts are also classified on the content into serous, chylous, hemorrhagic and chylolymphatic cysts.

These cysts may be asymptomatic and have variable clinical presentation including abdominal distension, abdominal lump. These may be present as complications including intestinal obstruction, hemorrhage, infection, rupture, volvulus and obstruction of the urinary or biliary tract. It was presented as intestinal obstruction in our case.

These may occur in the any part of mesentery. Theses cysts most commonly involve the small bowel mesentery (ileum in $60 \%$ ) and mesocolon (ascending colon in 40\%). It was presented in ileal mesentery in our case.

Mesenteric cysts are palpable in the $50 \%$ of cases and mobile transversely and not longitudinally [3]. While in our case it was nonpalpable.

A plan abdominal radiograph shows homogenous mass with displacing the bowel around it. While in cases of intestinal obstruction multiple air fluid levels. Ultrasonography abdomen is the investigation of choice. It shows 'fluid-fluid level' in cases of the chylolymphatic cysts. this is visible due to formation of the an upper fluid level by lighter chyle over a lower fluid level of heavier chyle. Contrast enhanced computed tomography shows presence of fluid levels of different echodensities, relationships with the bowel and other vital structures. Antenatal ultrasonography also detect in the fetus. While in our case presentation was acute intestinal obstruction, so we performed only x-ray abdomen. It showed multiple air fluid level, so we explored in emergency. We didn't perform ultrasonography and computed tomography.

Mesenteric cyst may be uni or multilocular and filled with serousfluid or chyles [4]. There are thin walled and have incomplete lining epithelium composed of endothelial cells. There don't have mucosa or muscular wall. In our case it was filled with serous fluid and chyle with multilocular.

The complete excision with or without resection of the involved bowel is the treatment of the choice. It has an excellent prognosis and no recurrence have been reported. Laparoscopic excision has been successful in some cases; however it is difficult to perform in the larger cyst just like our case. Marsupialization and drainage are also part of the treatment, but these have high recurrence rate $[4,5]$.

\section{CONCLUSION}

Chylolymphatic cysts are rare types of mesenteric cysts, but it should be kept in mind as one of the differential diagnosis. Complete excision with or without involved bowel is the treatment of choice and has excellent prognosis.

\section{CONFLICT OF INTEREST}

Declared none.

\section{ACKNOWLEDGEMENTS}

Declared none.

\section{REFERENCES}

[1] Rattan KM, Nair VJ, Pathak M, Kumar S. Pediatric chylolymphatic mesenteric cyst- a separate entity from cystic lymphangiomas: A case series. J Med Case Rep 2009; 3: 111. DOI: 10.1186/1752-1947-3-111

[2] Muramori K, Zaizen Y, Noguchi S. Abdominal lymphangioma in children: Report of three cases. Surg Today 2009; 39: 414-7. DOI: $10.1007 / \mathrm{s} 00595-008-3854-\mathrm{z}$

[3] Al-Haifi MB, Abdulsmad AM, Juma TH. Laparoscopic excision of mesenteric cyst: Case report. Kuwait Med J 2007; 39: 167-9.

[4] Barut I, Tarhan OR, Ciris M, Akdeniz Y, Bulbul M. Intestinal obstruction due to a mesenteric cyst. Yunsei Med J 2004; 45: 256-8. DOI: 10.3349/ymj.2004.45.2.356

[5] Chang TS, Richard R, Abramowksy CR, et al. Mesenteric cystic masses: A series of 21 pediatric cases and review of the literature. Fetal Pediatr Pathol 2011; 30: 40-4. DOI: $10.3109 / 15513815.2010 .505623$ 